\title{
Evaluación de la primera alimentación en larvas de capaz Pimelodus grosskopfii bajo condiciones de laboratorio
}

\section{Evaluation of first feeding of capaz larvae Pimelodus grosskopfii under laboratory conditions}

\author{
Rubén Valbuena $\mathrm{V}^{1}$ M.Sc, Beatriz Zapata-Berruecos, ${ }^{1}$ Esp, \\ Angélica Otero-Paternina, ${ }^{2}$ M.Sc.
}

\begin{abstract}
${ }^{1}$ Universidad Surcolombiana, Facultad de Ciencias Exactas y Naturales, Av Pastrana Borrero Carrera 1A, Neiva, Colombia. ${ }^{2}$ Universidad Estadual Paulista, Câmpus de São José do Rio Preto, Brasil. *Correspondencia: angelicaoterop@gmail.com
\end{abstract}

Recibido: Julio de 2011; Aceptado: Agosto de 2012.

\section{RESUMEN}

Objetivo. Evaluar el desempeño y sobrevivencia de larvas de capaz Pimelodus grosskopfii suministrando alimento vivo (Cladóceros, Copépodos y Artemia salina). Materiales y métodos. Larvas de capaz fueron ubicadas en recipientes plásticos con un volumen útil de $3 \mathrm{~L}$, a una densidad de 10 larvas $L^{-1}$, fueron alimentadas cuatro veces al día, durante 15 días con nauplios de Artemia recién eclosionadas, Cladóceros de los géneros Moina y Ceriodaphnia y Copépodos calanoides. Las larvas de capaz se pesaron y se midieron al inicio y al final del experimento para estimar ganancia en peso (GP), ganancia en longitud (GL), tasa de crecimiento específico (TCE), Factor de crecimiento relativo (FCR) y sobrevivencia (S) Resultados. El tratamiento que presentó los mejores resultados en GP, GL y $S$ fue el de larvas alimentadas con nauplios de Artemia $(3.8 \pm 0.2 \mathrm{mg}, 8 \pm 0.7 \mathrm{~mm}$ y $48.3 \%$ respectivamente) seguido de los tratamientos donde adicionó cladóceros y copépodos Conclusiones. Los nauplios de Artemia fue el tratamiento que presentó los mejores resultados en las variables productivas evaluadas en larvas de $P$. grosskopfii al inicio de su alimentación exógena.

Palabras clave: Artemia, Cladóceros, Copépodos, Pimelodus grosskopfii (Fuente: AIMS).

\section{ABSTRACT}

Objective. Evaluate the performance and survival of capaz larvae Pimelodus grosskopfii after supplying live food (Cladocera, Copepod and Artemia Salina). Materials and methods. Capaz larva were placed in plastic containers with an useful volume of $3 \mathrm{~L}$, and a larvae density of $10 \mathrm{~L}^{-1}$, they were fed four times a day during 15 days with newly hatched Artemia nauplii, Cladocerans of the genders Moina and Ceriodaphnia and Copepods calanoides. Capaz larva were weighed and measured at the beginning and the end of the experiment in order to evaluate their weight gain (WG), length gain (LG), specific growth rate (SGR), relative growth factor (RGF), and survival rate(S). Results. The treatment that showed the best results in WG, LG and $S$ was the lot fed with Artemia nauplii $(3.8 \pm 0.2 \mathrm{mg}, 8 \pm 0.7 \mathrm{~mm}$ and $48.3 \%$ respectively), followed by sample supplied with Cladocera and Copepods. Conclusions. The Artemia nauplii treatment revealed the best results on the productive variables evaluated in $P$. grosskopfii larvae at the beginning of their exogenous feeding.

Key words: Artemia, Cladocerans, Copepods, Pimelodus grosskopfii (Source: AIMS). 


\section{INTRODUCCIÓN}

Las investigaciones colombianas relacionadas con acuicultura pretenden desarrollar paquetes tecnológicos para incorporar especies de silúridos y diversificar la piscicultura nacional. El capaz (Pimelodus grosskopfii) por sus características de calidad de la carne (blanca y ausencia de espinas intramusculares), tiene una alta aceptación comercial, características que junto a su potencial reproductivo mostrado en ensayos preliminares (1), lo hace una especie promisoria para el desarrollo de tecnologías de cultivo y a través de su vinculación a la piscicultura lograr la diversificación y ampliación de la oferta nacional. Esta especie se encuentra distribuida en las cuencas de los ríos Magdalena, Cauca y San Jorge (2), así como en el embalse de Betania (Huila) (3); y es considerada de interés comercial para la cuenca del río Magdalena (4).

Uno de los mayores problemas en la producción de alevinos de estas especies nativas es la fase de larvicultura, en las que se ha observado altas tasas de mortalidad, siendo las causas más relevantes: canibalismo, comportamiento derivado de sus hábitos piscívoros, condiciones ambientales inadecuadas y poco conocimiento de su hábitat y hábitos alimenticios en sistemas naturales $(5,6)$. Por eso es importante el levante de larvas y posterior alevinaje con la utilización de organismos vivos como fuente de alimento que sustenta el crecimiento y el éxito de esta fase (7). El suministro de estos organismos ofrece ventajas principalmente por el valor nutritivo el cual se basa en el contenido de aminoácidos y ácidos grasos esenciales, entre otros elementos que favorecen el crecimiento y la sobrevivencia de las larvas (8).

El objetivo del presente trabajo fue evaluar el desempeño y sobrevivencia de larvas de capaz suministrando alimento vivo.

\section{MATERIALES Y MÉTODOS}

Localización y descripción del área de estudio. La investigación se llevó a cabo en el Laboratorio de Alimento Vivo de la estación Piscícola del Alto Magdalena adscrita al Instituto Colombiano para el Desarrollo Rural - INCODER. El clima se caracteriza por una altura promedio de 930 m.s.n.m, temperatura de $24^{\circ} \mathrm{C}$, precipitación pluvial anual de $1250 \mathrm{~mm}$.

Material biológico. Las larvas de capaz Pimelodus grosskopfii fueron obtenidas por reproducción artificial, usando extracto de hipófisis de carpa, siguiendo los protocolos evaluados para esta especie por Valbuena et al (1). La eclosión ocurrió entre las 18-20 horas post fertilización con una temperatura del agua promedio de $24.4 \pm 0.4^{\circ} \mathrm{C}$.

Toma de muestras. En la fase larvaria se tomaron muestras a partir de las 0 horas post eclosión (HPE); las muestras se tomaron cada hora hasta las 16 HPE y cada cuatro hasta las 80 HPE con un $n=$ de 10 larvas por muestreo. Las larvas se fijaron en formol tamponado al $4 \%$ $(\mathrm{v} / \mathrm{v})$. Posteriormente, con una reglilla adaptada a un estereoscopio (Nikon SMZ800, Japón) se realizaron mediciones al saco vitelino $(\mathrm{mm})$, abertura de la boca y longitud total $(\mathrm{mm})$ para determinar el volumen y reabsorción del saco vitelino así como también la abertura máxima de la boca, empleando las siguientes formulas:

Volumen del saco. Para el volumen se determinó la altura y longitud del vitelo, aplicando la fórmula descrita por Junqueira (9)

V $\frac{\pi}{6} * L \quad H^{2}$

Donde:

$\mathrm{V}$ : volumen del saco vitelino expresado en $\mathrm{mm}^{3}$ L: longitud total del saco vitelino expresada en $\mathrm{mm}$

$\mathrm{H}$ : altura del saco vitelino expresada en $\mathrm{mm}$.

Determinación de la reabsorción del saco vitelino. Para determinar el porcentaje de reabsorción del saco se empleó la siguiente fórmula

$R S V=V m * \frac{100}{V i}$

Donde:

$\mathrm{Vm}$ : volumen del saco vitelino $\left(\mathrm{mm}^{3}\right)$ a la hora de muestreo.

Vi: volumen del saco vitelino $\left(\mathrm{mm}^{3}\right)$ de larvas recién eclosionadas.

Determinación de la abertura máxima de la boca (AMB). Para determinar la abertura máxima de la boca se consideró un ángulo de $90^{\circ}$ mediante la siguiente ecuación (10).

$A M B=L M S * \sqrt{2}$

Donde:

LMS: longitud máxima del maxilar superior en micras.

Evaluación de variables productivas en larvas de capaz alimentadas con diferentes tipos de organismos vivos. Larvas de $30 \mathrm{HPE}$ 
fueron distribuidas en recipientes plásticos con capacidad util de $3 \mathrm{~L}$, cada recipiente contenían 30 larvas para una densidad de 10 larvas $L^{-1}$ y se mantubo con aireación constante. Antes de iniciar el experimento se permitió un día de adaptación a las condiciones experimentales y las larvas muertas fueron remplazadas con larvas del mismo desove para mantener la densidad anotada. Los tratamientos fueron: nauplios de Artemia recién eclosionados, Cladóceros de los géneros Moina y Ceriodaphnia y Copépodos del genero Diaptomus, con cuatro réplicas por tratamiento. Los organismos zooplanctónicos utilizados en este ensayo provenían de cultivos.

Las larvas fueron alimentadas con una frecuencia de cuatro raciones diarias, suministradas a las 07:00, 12:00, 16:00 y 20:00 horas. La cantidad de alimento suministrado fue de 10 organismos por larvas en cada ración, durante los primeros 5 días. Esta se duplicó en los siguientes 5 días y se triplicó hasta el final del ensayo; por volumetría se cuantificaban los organismos a suministrar. Dos horas después de cada ración se realizó limpieza a cada recipiente con el objetivo de retirar los organismos que no fueron consumidos. El ensayo fue conducido bajo condiciones controladas de laboratorio, temperatura de $27 \pm 1^{\circ} \mathrm{C}$ en oscuridad total y tuvo una duración de 15 días.

Determinación del crecimiento y sobrevivencia larval. Al inicio y final del ensayo, las larvas de cada tratamiento fueron pesadas individualmente en una balanza analítica (Pioneer OHAUS, China) y medidas con un calibrador $( \pm 0.01)$ con el fin de determinar los siguientes parámetros productivos:

\section{Ganancia en peso $(\mathrm{mg})$.}

$G P=P f-P i$

Donde:

Pf: peso final

$\mathrm{Pi}$ : peso inicial

\section{Ganancia en longitud ( $\mathbf{m m})$.}

$G L=L f-L i$

Donde:

Lf: longitud final

Li: longitud inicial

\section{Sobrevivencia (\%).}

$\mathrm{S}=\frac{N f}{\mathrm{Ni}} * 100$

Donde:

$\mathrm{Nf}$ : número de larvas finales

$\mathrm{Ni}$ : número de larvas iniciales

\section{Ganancia diaria de peso ( $\left.\mathrm{mg} \mathrm{día}^{-1}\right)$.}

$$
G D P=\frac{P f-P i}{T}
$$

Donde:

Pf: Peso final

Pi: Peso inicial

T: días del ciclo de producción

Tasa de crecimiento específica.

$$
T C E=\frac{\operatorname{LnPf-\operatorname {LnP}i}}{T} * 100
$$

Factor de crecimiento relativo.

$$
F C R=\frac{P f}{L f}
$$

Donde:

Pf: Peso final

Lf: Longitud final

En cada unidad experimental una vez al día se realizaron mediciones de los siguientes parámetros de calidad de agua: temperatura $\left({ }^{\circ} \mathrm{C}\right)$, oxígeno disuelto $\left(\mathrm{mg} \mathrm{L}^{-1}\right)$ y $\mathrm{pH}$ haciendo uso de una sonda multiparamétrica YSI (YSI Professional Plus, USA).

Análisis estadístico. Los datos de las variables productivas se presentan mediante estadística descriptiva. Para analizar los efectos del tipo de alimento vivo se realizó un análisis de varianza (ANOVA) de una vía tipo MLG, verificando previamente los supuestos de normalidad (prueba de Kolmogorov-Smirnov) y homogeneidad de varianzas (prueba de Levene). Se utilizó la prueba de Bonferroni con el fin de comparar las medias entre los diferentes tratamientos. El criterio de significancia fue $p<0.05$. Los datos de sobrevivencia fueron transformados con la función del Arcoseno e igualmente la ganancia de peso fue trasformada usando la función logaritmo natural. Los análisis estadísticos fueron realizados usando el programa $\mathrm{R}$.

\section{RESULTADOS}

Los parámetros de calidad de agua, determinados durante el ensayo, se muestran en la tabla 1. La temperatura, oxígeno disuelto y $\mathrm{pH}$ fueron uniformes en todos los tratamientos durante los 15 días del ensayo, sin presentar diferencias significativas entre los tratamientos $(p>0.05)$.

El volumen inicial del saco vitelino en las larvas fue de $0.154 \mathrm{~mm}^{3}$; este fue reabsorbido a las 50 HPE aproximadamente. 
Tabla 1. Parámetros de calidad de agua monitoreados en los diferentes tratamientos, durante la evaluación del desempeño del capaz (Pimelodus grosskopfii) en los ensayos de primera alimentación. Valores expresados como media \pm SD.

\begin{tabular}{cccc}
\hline Tratamientos & $\begin{array}{c}\text { Temperatura } \\
\left({ }^{\circ} \mathbf{C}\right)\end{array}$ & $\begin{array}{c}\text { Oxígeno } \\
\text { Disuelto }(\mathbf{m g} \\
\left.\mathbf{L}^{-1}\right)\end{array}$ & $\mathbf{p H}$ \\
\hline Artemia & $24.9 \pm 1.2$ & $4.7 \pm 0.4$ & $7.9 \pm 0.2$ \\
Cladóceros & $24.5 \pm 1.1$ & $4.4 \pm 0.6$ & $8.1 \pm 0.3$ \\
Copépodos & $24.6 \pm 1.1$ & $4.5 \pm 0.7$ & $8.2 \pm 0.3$ \\
\hline
\end{tabular}

Se observó abertura de la boca a las $11 \mathrm{HPE}$, alcanzando el máximo a las $43 \mathrm{HPE}$ con un valor de $600 \pm 0.60 \mu \mathrm{m}$. Las larvas iniciaron la alimentación exógena entre las 40 y $45 \mathrm{HPE}$, con una longitud de $4.26 \pm 0.3 \mathrm{~mm}$ Lt $y$ un peso de $1 \pm 0.5 \mathrm{mg}$.

Se observaron diferencias significativas en las medias de longitud total $(p<0.05)$ y peso corporal $(p<0.05)$ de las larvas sometidas a los diferentes tratamientos durante $15 \mathrm{~d}$. El tratamiento que presentó la mayor ganancia de peso y longitud fue el de larvas alimentadas con Artemia con valores de $15.3 \pm 4.8 \mathrm{mg}$ y $13.6 \pm 1.6$ $\mathrm{mm}$ respectivamente; el que presentó el menor valor fue el tratamiento de larvas alimentadas con copépodos $(3.8 \pm 0.2 \mathrm{mg}$ y $8 \pm 0.7 \mathrm{~mm}$, respectivamente) (Figuras 1 y 2 ).

En cuanto al porcentaje de sobrevivencia se presentaron diferencias significativas entre los tratamientos $(p<0.05)$. La mayor sobrevivencia se obtuvo en el tratamiento donde se suministraron nauplios de Artemia recién eclosionados con un valor de $48.3 \%$, seguido del tratamiento donde se alimentó con cladóceros con el $6.7 \%$ (Figura 3 ).

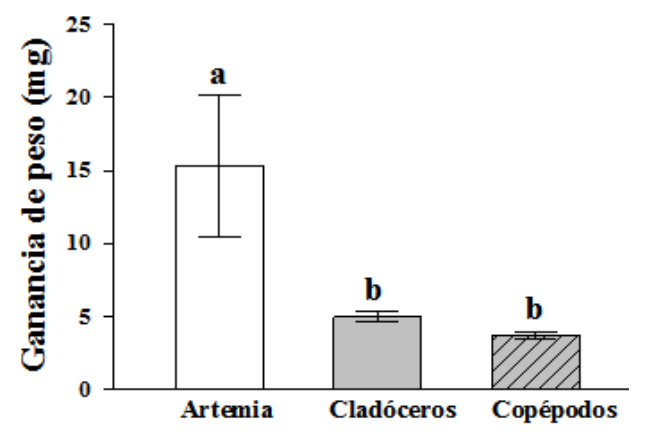

Figura 1. Ganancia de peso de larvas del capaz (Pimelodus grosskopfii). Valores mostrados como media $\pm S D$. Diferencias entre los tratamientos son indicados como $a$, b según el test de Bonferroni $(p<0.05)$.

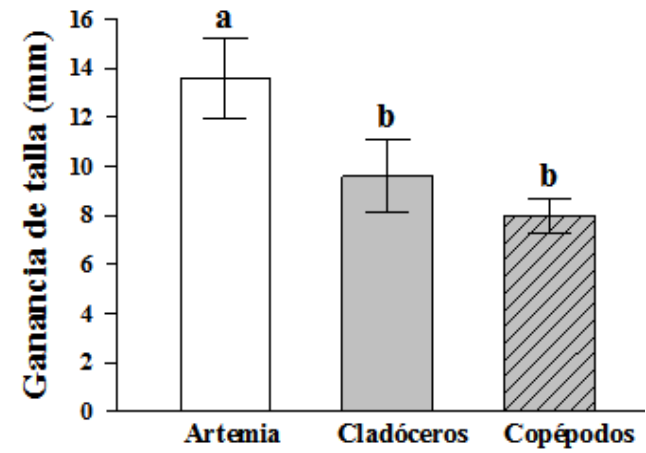

Figura 2. Ganancia de longitud en larvas del capaz utilizando tres diferentes fuentes de alimento vivo. Valores mostrados como media \pm SD. Diferencias entre los tratamientos son indicados como a, b según el test de Bonferroni $(p<0.05)$.

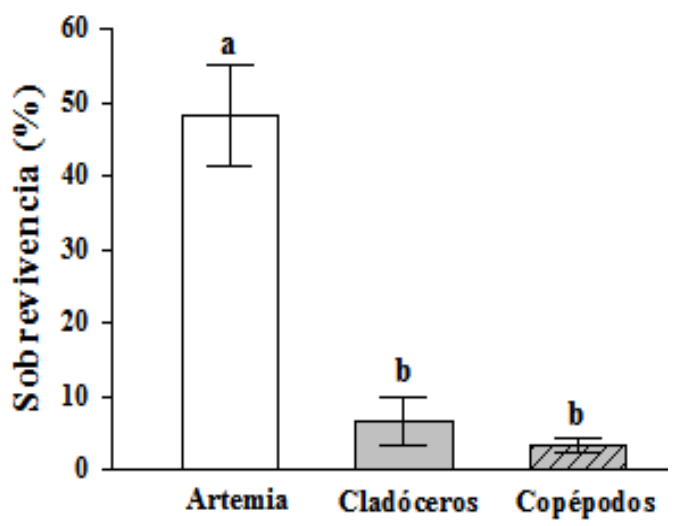

Figura 3. Porcentaje (\%) de sobrevivencia de larvas del capaz, utilizando tres diferentes fuentes de alimento vivo. Valores mostrados como media SD. Entre columnas, para cada tratamiento, letras diferentes indican diferencias significativas $(p<0.05)$ según el test Bonferroni.

En la tabla 2 se muestran los promedios de ganancia diaria de peso, tasa de crecimiento específico y el factor de crecimiento relativo. Los mayores valores para estas variables productivas se presentaron en el tratamiento donde se suministró nauplios de Artemia.

Tabla 2. Variables productivas determinadas en larvas del capaz utilizando tres diferentes fuentes de alimento vivo. Valores mostrados como media \pm SD.

\begin{tabular}{cccc}
\hline Tratamientos & $\begin{array}{c}\text { Ganancia diaria } \\
\text { de peso } \\
\text { (mg/día) }\end{array}$ & $\begin{array}{c}\text { Factor de } \\
\text { crecimiento } \\
\text { relativo }\end{array}$ & $\begin{array}{c}\text { Tasa de } \\
\text { crecimiento } \\
\text { específico }\end{array}$ \\
\hline Artemia & $1.02 \pm 0.32$ & 1.1 & 18.2 \\
Cladóceros & $0.32 \pm 0.02$ & 0.4 & 10.5 \\
Copépodos & $0.25 \pm 0.01$ & 0.5 & 8.9 \\
\hline
\end{tabular}




\section{DISCUSIÓN}

Las características físicas y químicas del agua durante la etapa de larvicultura requieren de un monitoreo continuo para garantizar un buen desempeño. Dentro de las variables de importancia, el oxígeno disuelto es una de las más críticas en el cultivo de organismos acuáticos. Su deficiencia incide sobre variables como ganancia de peso, conversión alimenticia y sobrevivencia (10).

Los valores de oxígeno en este estudio fueron de $4.5 \pm 0.2$, según Link et al (11) y Nuñez et al (12) los valores de oxígeno disuelto se deben mantener por encima de los $4 \mathrm{mg} \mathrm{L}^{-1}$ en la fase de larvicultura. Los resultados en este estudio se encuentran dentro de este rango. Los valores de $\mathrm{pH}$ fueron de $8.07 \pm 0.2$, según Atencio-García et al (10), el pH en las especies neotropicales debe mantenerse entre 6 y 9. Los valores de temperatura oscilaron en promedio de $24.7 \pm 0.2$, los cuales se encuentran por debajo a lo reportado en otros silúridos como $P$. fasciatum $(13,14)$ y Leiarius marmoratus (15). La temperatura está asociada directamente con el metabolismo de los organismos y juega un papel importante durante el desarrollo desde la fertilización hasta su eclosión y posteriores estadios $(12,16)$.

Una de las características que se debe tener en cuenta para las buenas prácticas de primera alimentación es el momento en el cuál se va a suministrar el primer alimento, el cual tiene una relación directa con la reabsorción del saco vitelino y el tamaño de la boca. La abertura máxima de la boca en larvas de capaz para este estudio fue de $600 \pm 0.60 \mu \mathrm{m}$, similar a lo reportado en otras especies de bagres como blanquillo (Sorubim cuspicaudus) con una abertura de $600 \mu \mathrm{m}$ (17); Ramírez et al (15) reportaron para yaque $L$. marmoratus un valor de $532.6 \pm 7.8 \mu \mathrm{m}$ el cual se encuentra por debajo a lo reportado en este estudio, mientras que Atencio et al (18) reportaron para dorada (Brycon sinuensis) la abertura bucal máxima en $1.2 \pm 0.1 \mathrm{~mm}$ y en bocachico Prochilodus magdalenae de $671 \pm$ $12.8 \mu \mathrm{m}$ (10). La abertura bucal al inicio de la alimentación exógena determina la cantidad y el tipo de presa que puede ser ingerido sumado a otras características morfológicas asociadas al aparato mandibular (19). La alimentación exógena se inició entre las 40 y $45 \mathrm{HPE}$, valores similares a lo reportado en $S$. cuspicaudus las cuales fueron de 46 a 48 HPE (16) y 44 a 48 HPE en $P$. magdalenae (10), mientras para $L$. marmoratus fue de 35 y 40 HPE (15), Atencio-García et al (20) registró para yamú Brycon siebenthalae la alimentación exógena a las $36 \mathrm{HPE}$.
Dentro de los tratamientos las larvas alimentadas con nauplios de Artemia presentaron los mejores resultados en cuanto a ganancia de peso y longitud, resultados similares se han observado en los procesos de larvicultura de otros bagres como P. fasciatum (13), Rhamdia sebae (21), el bagre asiático Pangasius bocourti (22). Sin embargo, para la especie $L$. marmoratus se reportan ganancias menores (15). Se ha determinado que para la mayoría de silúridos en su etapa larval, se requiere de $50 \%$ de proteína bruta y entre 10 y $15 \%$ de lípidos. Artemia ha sido utilizada debido a sus aportes nutricionales, la cual contiene ácidos grasos ( $n-3$ y n-6) y más del $47 \%$ de proteína (23). Sin embargo, sus elevados costos obligan a explorar otras fuentes de organismos zooplanctónicos. Dentro de estos, los cladóceros se presentan como alternativa alimenticia en la larvicultura de silúridos, estudios muestran un aumento significativo en el crecimiento de larvas $P$. fasciatum alimentadas con cladóceros sin enriquecer (13). De igual manera la mayor sobrevivencia se presentó en el tratamiento donde se suministró nauplios de Artemia, seguida por el tratamiento de cladóceros; resultados similares obtuvieron en L. marmoratus (15), Rhamdia sebae (21), P magdalenae (10). Nuñez et al (12) reportan que larvas de $P$. fasciatum alimentadas con zooplancton natural y Artemia, presentaron altas tasas de sobrevivencia, hasta los 15 días posteclosión (DPE) luego decrece considerablemente en los tratamientos alimentados con zooplancton natural, debido a que el zooplancton tiene baja digestibilidad de nutrientes y contenido de energía $(24,25)$.

Con respecto a las variables productivas ganancia diaria de peso, tasa de crecimiento y factor de crecimiento, los mejores resultados nuevamente se presentaron en el tratamiento con Artemia seguido del tratamiento de cladóceros; resultados similares a lo reportado en $P$. fasciatum (26) $S$. cuspicaudus cuando se le suministró mesocosmo compuesto por cladóceros y copépodos (27). Estos resultados quizás se debieron al tamaño del estadio de nauplio, el cual es apto para muchas larvas de peces en la larvicultura comercial (22).

En este estudio se detectó canibalismo en todos los tratamientos, durante los primeros días del ensayo, lo cual coincide con lo reportado en $B$. sinuensis (18), L. marmoratus (15), $P$. fasciatum (14). Según Atencio-García et al (5), el canibalismo es una conducta adaptativa en condiciones de escasez de alimento, la cual está definida por la frecuencia de alimentación así como por la distribución y tamaño del alimento. 
Los resultados del presente estudio permiten concluir que los nauplios de Artemia por su tamaño y composición bromatológica fue el mejor alimento para larvas de $P$. grosskopfii al inicio de su alimentación exógena, lo cual se reflejó en las variables que fueron evaluadas, seguido de las larvas que fueron alimentadas con cladóceros. Se recomienda seguir evaluando otros tipos de alimento para consolidar el proceso de larvicultura de esta especie.

\section{Agradecimientos}

Al Ministerio de Agricultura y Desarrollo Rural, Universidad Surcolombiana y a la Corporación Centro De Desarrollo Tecnológico Piscícola Surcolombiano - Acuapez (convenio No 054) por el apoyo financiero, así como al Instituto Colombiana de Desarrollo Rural - INCODER por el apoyo logístico y a los Tecnólogos en Acuicultura Yurany Perdomo y Daiver Rojas, por su colaboración en la conducción del ensayo.

\section{REFERENCIAS}

1. Valbuena-Villarreal RD, Zapata-Berruecos BE, Cruz-Casallas PE. Reproducción inducida de Capaz (Pimelodus grosskopfii) con extracto de hipófisis de carpa: reporte preliminar. Revista Orinoquia 2010; 14 (2):133-139.

2. Maldonado-Ocampo JA, Vari RP, Usma JS. Checklist of the freswater fishes of Colombia. Biota Colombiana 2008; 9(2): 143-237.

3. Villa-Navarro FA. Diferenciación entre poblaciones de Pimelodus clarias y Pimelodus grosskopffi (Siluriformes: Pimelodidae) en la cuenca del río Magdalena Colombia. [Tesis de Maestría]. Cali, Colombia: Universidad del Valle; 2002.

4. Instituto Colombiano de Desarrollo Rural - INCODER y Corporación Colombia Internacional. Pesca y Acuicultura. [Informe técnico]. Bogotá, Colombia; 2006.

5. Atencio-García VJ, Zaniboni-Fhilo E. El canibalismo en la larvicultura de peces. Rev MVZ Córdoba 2006; 11(1): 9-19

6. Kestemont $\mathrm{P}$, Jourdan $\mathrm{S}$, Houbart $\mathrm{C}$, Paspatis M, Fontaine $P$, Cuvier A, Kentouri $M$, Baras E. Size heterogeneity, cannibalism and competition in cultured predatory fish larvae: biotic and abiotic influences. Aquacult 2003; 227: 333-356.

7. Chuan Lim L, Dhert P, Sorgeloos P. Recent developments in the application of live feeds in the freshwater ornamental fish culture. Aquacult 2003; 227:319-331.
8. Sipaúba-Tavares LH, Rocha O. Produção de Plâncton (fitoplâncton e Zooplâncton) para alimentação de Organismos Aquáticos. $1^{\text {ra }}$ ed. São Carlos: RIMA; 2003.

9. Junqueira CAP. Efeito da temperatura no desenvolvimento inicial de larvas de "Curimbatá" Prochilodus scrofa Steindachner, 1882 (Characiformes, Prochilodontidae). [Tesis Maestría]. Santa Catarina, Brasil: Universidade Federal Santa Catarina; 1999.

10. Atencio-García $V$, Kerguelén $E$, Wadnipar L, Narváez A. Manejo de la primera alimentación del bocachico (Prochilodus magdalenae). Rev MVZ Córdoba 2003; 8(1):254-260.

11. Link de Rosso F, Bolner K, Baldisserotto B. Ion fluxes in silver catfish (Rhamdia quelen) juveniles exposed to different dissolved oxygen levels. Neotrop Ichthyol 2006; 4(4):435-440.

12. Nuñez J, Duque R, Corcuy-Arana N, Duponchelle F, Renno J, Raynaud T. Induced breeding and larval rearing of Surubí, Pseudoplatystoma fasciatum (Linneaus, 1766), from the bolivian Amazon. Aquac Res 2008; 39:764-776.

13. Marciales-Caro LJ, Díaz-Olarte JJ, MedinaRobles VM, Cruz-Casallas PE. Evaluación del crecimiento y sobrevivencia de larvas de bagre rayado Pseudoplatystoma fasciatum (Linneaus, 1766) alimentadas con alimento vivo natural y enriquecido con ácidos grasos. Rev Colomb Cienc Pecu 2010; 23:308-316. 
14. Díaz-Olarte JJ, Cruz-Casallas NE, MarcialesCaro LJ, Medina-Robles VM, Cruz-Casallas PE. Efectos de la densidad de siembra y disponibilidad de alimento sobre el desarrollo y sobrevivencia de larvas de Pseudoplatystoma fasciatum. Revista Orinoquia 2009; 13 (1): 21-30.

15. Ramírez-Merlano JA, Otero-Paternina AM, Corredor-Santamaría W, Medina-Robles VM, Cruz-Casallas PE, Velasco-Santamaría, YM. Utilización de organismos vivos como primera alimentación de larvas de yaque (Leiarius marmoratus) bajo condiciones de laboratorio. Revista Orinoquia 2010; 14(1):45-58.

16. Pereira C, Barcellos L, Kreutz L, Quevedo R, Ritter F, Silva L. Embryonic and larval development of jundiá (Rhamdia quelen, Quay \& Gaimard, 1824, pisces, Teleostei), a South American catfish. Braz J Biol 2006; 66(4):1057-1063.

17. Novoa JD, Cataño Y. Descripción del desarrollo embrionario y larvario del blanquillo Sorubim cuspicaudus (Littmann, Burr y Nass, 2000). [Tesis de pregrado]. Montería, Colombia: Universidad de Córdoba; 2005.

18. Atencio García VJ, Pertuz Buelvas VM, Pérez Espitia F, Ortiz Mestra R, Pardo Carrasco SC. Manejo de la primera alimentación de dorada Brycon sinuensis ofreciendo larvas de bocachico Prochilodus magdalenae. Rev Colomb Cienc Pecu 2010; 23:317-324.

19. Meza-González OR, Benítez-Flores J, Paredes-Dominguez BM, González-Valle M. Descripción histológica del sistema digestivo en larvas de Chirostoma humboldtianum en la primera alimentación exógena. CIVA $2002 ; 313-322$.

20. Atencio-García V, Zaniboni-Fhilo E, PardoCarrasco S, Arias-Castellanos A. Influência da primeira alimentação na larvicultura e alevinagem do yamú Brycon siebenthalae. Acta Scientarium 2003; 25:61-72.
21. Muñoz F, Tobar JM, Arias JA. Respuesta a la primera alimentación en larvas de barbilla Rhamdia sebae C.F. (Pisces: Siluriformes, Pimelodidae). Revista Biotecnología en el Sector Agropecuario y Agroindustrial 2007; 5(1):47-93.

22. Hung L, Tuan N, Cacot P, Lazard J. Larval rearing of the Asian Catfish, Pangasius bocourti (Siluroidei, Pangasiidae): alternative feeds and weaning time. Aquacult 2002; 212:115- 127.

23. Sorgeloos P, Dhert A, Candreva P. Use of the brine shrimp, Artemia spp., in marine fish larviculture. Aquacult 2001; 200:147-159.

24. Baras $E$, Jobling M. Dynamics of intracohort cannibalism in cultures fish. Aquacult Res $2002 ; 33: 461-479$.

25. Baras E, Kestemont P, Melard C. Effect of stocking density on the dynamics of cannibalism in sibling larvae of Perca fluviatilis under controlled conditions. Aquacult 2003; 219:241-255.

26. Cubillos YD. Evaluación del suministro de organismos vivos como primera alimentación de larvas de bagre rayado Pseudoplatystoma fasciatum, bajo condiciones laboratorio. [Tesis de pregrado]. Fusagasugá, Colombia: Universidad de Cundinamarca; 2010.

27. Hernández-Bedoya JP, Gómez C, PrietoGuevara MJ, Atencio-García VJ, PardoCarrasco SC. Larvicultura del blanquillo Sorubim cuspicaudus alimentados con tres tipos de presas vivas En: IV Congreso Colombiano de Acuicultura. Medellín: Universidad de Antioquia: 2008. 